\title{
The long and the short of riboswitches
}

\author{
Alexander Serganov \\ Structural Biology Program, Memorial Sloan-Kettering Cancer Center, New York, NY, 10021, USA
}

\begin{abstract}
Regulatory mRNA elements or riboswitches specifically control the expression of a large number of genes in response to various cellular metabolites. The basis for selectivity of regulation is programmed in the evolutionarily conserved metabolite-sensing regions of riboswitches, which display a plethora of sequence and structural variants. Recent X-ray structures of two distinct SAM riboswitches and the sensing domains of the $\mathrm{Mg}^{2+}$, lysine, and FMN riboswitches have uncovered novel recognition principles and provided molecular details underlying the exquisite specificity of metabolite binding by RNA. These and earlier structures constitute the majority of wide-spread riboswitch classes and, together with riboswitch folding studies, improve our understanding of the mechanistic principles involved in riboswitch-mediated gene expression control.
\end{abstract}

\section{Introduction}

Since the discovery of riboswitches and related regulatory mRNA elements, it has become evident that the sensing and gene controlling functions traditionally attributed to proteins can be performed by RNAs [1,2]. Recent intensive biochemical and biophysical research has increased the appreciation of riboswitches as universal genetic factors that adjust gene expression in response to various chemical cues in evolutionarily diverse species $[3,4]$. The adaptation of protein biosynthesis to environmental conditions is achieved by riboswitches through the direct sensing of small-molecule metabolites present in cells at elevated concentrations. Most known riboswitches are located in the 5'-untranslated regions of bacterial genes associated with cognate metabolites and are involved in regulating gene expression at the levels of transcription attenuation and translation initiation. The regulation is typically based on the interplay between alternative conformations within an evolutionarily conserved metabolite-sensing domain and a variable expression platform bearing gene control elements (Figure 1). In some bacteria, the riboswitch-based feedback regulatory circuits exert control over a significant portion of the genome, competing in number with metabolite-sensing regulatory proteins [3]. Several thiamine-pyrophosphate-(TPP)-sensing riboswitches have been also found in fungal and plant genes, where they modulate mRNA splicing and stability [5].

To date, riboswitches encompass more than a dozen classes specific to various types of metabolites. Not surprisingly, the metabolite-sensing domains of riboswitches demonstrate high diversity in composition, size, and complexity of their secondary and tertiary structures [6]. Principles underlying the exquisite selectivity of metabolite recognition became better understood after determination of the three-dimensional structures of guanine- [7,8], adenine-

corresponding author: serganoa@mskcc.org.

Publisher's Disclaimer: This is a PDF file of an unedited manuscript that has been accepted for publication. As a service to our customers we are providing this early version of the manuscript. The manuscript will undergo copyediting, typesetting, and review of the resulting proof before it is published in its final citable form. Please note that during the production process errors may be discovered which could affect the content, and all legal disclaimers that apply to the journal pertain. 
[8], TPP- [9,10], and $S$-adenosylmethionine (SAM)-[11] sensing domains and of the $g \operatorname{lmS}$ riboswitch/ribozyme [12,13]. During the last two years, this list has been extended by five new riboswitches whose X-ray structures range from simple conformations, such as a small pseudoknot, to large multi-helical folds. These recent structural advances along with studies of molecular mechanisms of riboswitch function will be primarily discussed herein, thus complementing earlier reviews on mRNA recognition by metabolites and proteins $[14,15]$.

\section{The expanding world of riboswitches}

After 2006, several new classes have been added to the existing collection of riboswitches responsive to adenine, guanine, TPP, SAM, flavin mononucleotide (FMN), adenosylcobalamin, lysine, glycine, and glucosamine-6-phosphate (GlcN6P) [3]. The most important discovery was the identification of the riboswitch specific for the second messenger cyclic di-guanosine monophosphate (di-GMP) in bacteria and bacteriophages [16••]. Cyclic di-GMP-sensing allows for the RNA-based control of genes that are responsible for wideranging physiological transformations within bacterial cells and are not directly involved in the metabolism of the compound. Furthermore, the riboswitch family receptive to purines and purine derivatives was expanded by two variants of the queuosine precursor preQ $_{1}$ sensor $[17,18 \bullet]$, the shortest known riboswitch that controls biosynthesis of the hypermodified nucleoside present in certain tRNAs, and by the 2'-deoxyguanosine sensor [19] that deviates from the classical adenine/guanine riboswitch sequences. In addition, SAM metabolism, dependent on five riboswitch variants in different bacteria [20], has been found to rely on yet another type of mRNA segment targeted by $S$-adenosylhomocysteine (SAH) [21]. Lastly, the first metallosensor in Salmonella enterica mgtA mRNA [22] has been complemented by the findings of a different magnesium sensor [23••] and RNA motifs triggered by molybdenum and related tungsten cofactors Moco and Tuco [24].

\section{New riboswitch architectures}

Despite the continuous success in identification of new riboswitch classes, the determination of riboswitch structures remains a tedious and time-consuming process. However, recent structural studies have succeeded in the determination of challenging riboswitch structures, varying in size and complexity.

Intriguingly, one of the large ribosensors, called M-box, is specific for $\mathrm{Mg}^{2+}$ cations, one of the smallest and most abundant cellular components [23]. The sensor controls $\mathrm{Mg}^{2+}$ homeostasis in Bacillus subtilis by attenuating the transcription of the $\mathrm{Mg}^{2+}$ transporter gene $m g t E$ (Figure 1a). The crystal structure (Figure 2a) revealed that the M-box architecture comprises a short stem-loop P6 and two parallel composite stems P1-P2 and P3-P4 connected by a three-way junction. The P3-P4 helix, from which stem P5 branches off, forms a closelypacked three-helical bundle P4/P5/P1-P2 stapled together by extensive tertiary contacts involving $\mathrm{J} 1 / 2, \mathrm{~L} 4$ and $\mathrm{L} 5$ regions. Unlike in other riboswitches, $\mathrm{Mg}^{2+}$ cations play a central role in folding the bridging region and mediating long-distance interactions that stabilize basepairing in the J1/2 region and in the adjacent P1 helix, thereby inducing the formation of the transcription terminator.

Since the structures of the M-box and other riboswitches are primarily built around three- or four-way helical junctions, they are difficult to use for characterization of more complex riboswitches, such as lysine and FMN riboswitches (Figure 1b, c), thereby demanding their structure determination. The structure of the $\sim 170 \mathrm{nt}$ Thermotoga maritima lysine-bound 'Lbox' domain, independently determined by two laboratories $[25 \bullet \cdot 26 \bullet \cdot$, consists of threehelical and two-helical bundles radiating from a compact five-way helical junction (Figure 2b). The P2/P2a/L2 stem-loop reverses its orientation through two $\sim 90^{\circ}$ turns near conserved motifs and is anchored by 'kissing' loop-loop interactions between L2 and L3. Parallel stems P2 and 
P4 are joined by a novel loop (L4)-helix (P2) contact. The junction is organized around the centrally positioned lysine that stabilizes the top region of the regulatory helix P1 in a way most similar to purine riboswitches.

The metabolite-sensing domain of the FMN riboswitch, an RFN element, presents further structural complexity. The structure of the $\sim 110 \mathrm{nt}$ Fusobacterium nucleatum RFN element [27••] (Figure 2c), one of the shortest among FMN riboswitches, has confirmed the six-way junction topology predicted by sequence analysis [3]. Unlike other large RNAs, the structure does not fold by collinear stacking of adjacent helices. Instead, it adopts a unique butterflylike scaffold, stapled together by two nearly identical domains P2-P6 and P3-P5 that are connected by the FMN-bound junction. The domains are related by the 2-fold symmetry typical for proteins and seldom observed in large RNAs. Each domain contains two stem-loop structures joined by T-loop and A-minor-like motifs, and shows a striking resemblance to a larger architectural module termed "T-loop PK domain," found in ribosomal RNA [28•]. The pseudo-symmetrical FMN does not take advantage of the riboswitch symmetry and orients its extremities towards different domains of the riboswitch. Such an arrangement is most similar to the TPP riboswitch $[9,10,29]$, although the FMN-binding regions are not as well separated. Contrary to other riboswitches, the alternative pairing in the FMN-sensing domain apparently involves its $5^{\prime}$ region, located much further from the complementary anti-terminator sequence than the $3^{\prime}$ segment transcribed later (Figure 1c). This atypical mechanism might be explained by a conserved substitution that weakens a tertiary contact and provides greater flexibility to the regulatory $\mathrm{J} 1 / 2$ sequence in the $5^{\prime}$ region.

The structures of the SAM-II/SAM- $\alpha[30 \bullet \bullet]$ and SAM-III/S $\mathrm{S}_{\mathrm{MK}}[31 \bullet \bullet]$ riboswitches, together with the earlier structure of the SAM-I/S-box riboswitch [11], uncover molecular details of evolutionarily independent and entirely distinct solutions for SAM sensing by RNA. Unlike the complex four-way-junction architecture of SAM-I riboswitch, the crystal structures of the $\sim 50 \mathrm{nt} \mathrm{SAM-II} \mathrm{and} \mathrm{SAM-III} \mathrm{riboswitches} \mathrm{revealed} \mathrm{simpler} \mathrm{compact} \mathrm{folds} \mathrm{based} \mathrm{on} \mathrm{a} \mathrm{H-type}$ pseudoknot and a three-way junction (Figure 2d, e).

The SAM-II structure from Sargasso Sea metagenome comprises a continuous helix P1/P2b/ P2a composed of three stacked helical elements, P1, P2a and P2b, and two loops, L1 and L3, which interact with the major and minor grooves of $\mathrm{P} 2 \mathrm{a} / \mathrm{P} 2 \mathrm{~b}$ and P1, respectively (Figure $2 \mathrm{~d}$ ). SAM positions itself inside the riboswitch and stabilizes the core of the RNA molecule. Since the Shine-Dalgarno (SD) sequence is intimately involved in SAM recognition, the SAM-II riboswitch likely represses translation via an occlusion mechanism rather than by more common secondary structure switching.

The Enterococcus faecalis SAM-III riboswitch folds into an inverted Y-shaped molecule, centered on the SAM-bound three-way junction (Figure 2e). A double-strand reversal in the $\mathrm{J} 3 / 2$ segment stabilizes the junction and forms a lid-like structure that constitutes part of the tight SAM-binding pocket. Complex formation shifts the conformational equilibrium towards the ligand-bound state with the SD sequence sequestered by contacts with the ligand and pairing within P1 and P4 helices (Figure 1d).

\section{Ligand binding by riboswitches: cations as ligands and as mediators of ligand recognition}

$\mathrm{Mg}^{2+}$ cations are essential for the folding and function of cellular RNAs, mostly due to their ability to shield negative charges and collapse RNA into a compact conformation. The TPP $[9,10,29,32]$ and $g \operatorname{lm} S$ riboswitches $[12,13]$ exploit this property to overcome the negative electrostatic character of the ligand phosphates. The M-box takes the neutralizing capability 
of $\mathrm{Mg}^{2+}$ cations a step further and uses them as sole ligands that affect gene expression through changes in riboswitch folding [23••].

The large $\mathrm{Mg}^{2+}$ sensor binds four $(\mathrm{Mg} 1-\mathrm{Mg} 4)$ out of six cations in the region involved in riboregulation [23••] (Figure 3a). These key cations apparently play non-equivalent roles in metallosensing. While $\mathrm{Mg} 1, \mathrm{Mg} 3$ and Mg4 stabilize the local conformations of L5, P2 and L4 regions, $\mathrm{Mg} 2$ bridges $\mathrm{L} 5$ and $\mathrm{P} 2 . \mathrm{Mg} 1$ forms 4 inner-sphere (one nucleobase and three sugarphosphate backbone) contacts with RNA, a number rarely observed in RNA structures, and, therefore, may crucially contribute to the tertiary interactions that facilitate pairing of the regulatory segment. Though other $\mathrm{Mg}^{2+}$ cations form less inner-sphere contacts with RNA, they may also be important for the specificity of metallosensing, given that almost all $\mathrm{Mg} 3$ and $\mathrm{Mg} 4$ outer-sphere interactions are made with nucleobases.

The high affinity interactions in the FMN-riboswitch complex also depend on magnesium and, to a lesser extent, on monovalent cations [27••]. As in TPP riboswitches [9,10,29], the extremities of FMN, the isoalloxazine ring system and the phosphate, provide the most important contacts with RNA while the middle ribityl moiety seems less critical for binding (Figure 3b). The ring system is sandwiched between purines, similar to the ligands in purine and TPP riboswitches [7-10,29], and is involved in specific hydrogen bonding with the nucleobase of conserved A99, reminiscent of purine, TPP and SAM-I riboswitches [7-11, 29]. The phosphate moiety forms the majority of hydrogen bonds with RNA, both direct and $\mathrm{Mg}^{2+}$-mediated. The $\mathrm{Mg}^{2+}$ cation can be substituted by $\mathrm{Ba}^{2+}, \mathrm{Mn}^{2+}, \mathrm{Ca}^{2+}$ and even by a [Co $\left.\left(\mathrm{NH}_{3}\right)_{6}\right]^{3+}$ group which mimics a fully-hydrated $\mathrm{Mg}^{2+}$ cation. Therefore, in contrast to the TPP riboswitch [32], neither the identity of the divalent cation nor the inner-sphere metal coordination with FMN and RNA are essential for FMN recognition by the riboswitch. Interactions with both the ring system and phosphate of FMN likely restrict the mobility of the $\mathrm{J} 6 / 1$ and $\mathrm{J} 1 / 2$ segments and contribute to the formation of the regulatory P1 helix.

Though the metal-dependent recognition of a phosphate-bearing FMN has been anticipated, the requirement of potassium for ligand recognition by a lysine riboswitch was unforeseen. Lysine is positioned in the middle layer of a tight pocket and is surrounded by evolutionarily conserved nucleotides (Figure 3c) [25••]. The 'main chain' and 'side chain' functionalities of the amino acid predominantly recognize nucleobases and the sugar-phosphate backbone, respectively. $\mathrm{A} \mathrm{K}^{+}$cation binds a carboxyl oxygen of lysine and zippers up the binding pocket through multiple coordination bonds, thereby explaining the severe impairment of ligand binding when $\mathrm{K}^{+}$is omitted or replaced by $\mathrm{Na}^{+}$. As in purine riboswitches $[7,8]$, the regulatory $\mathrm{P} 1$ helix is stabilized by contacts with the ligand and indirectly via tertiary interactions restricting mobility of the adjacent RNA segments, while discrimination against similar ligands is achieved by a combination of shape complementarity and specific hydrogen bonding.

\section{Ligand binding by riboswitches: same ligand, diverse recognition}

It was not entirely expected that SAM riboswitches would form very different binding pockets that interact with SAMs adopting distinct conformations. In the SAM-I structure (Figure 3d) [11], SAM assumes a compact U-shape conformation, wherein the methionine moiety stacks upon the adenine ring and is partially stabilized by an intramolecular $\pi$-cation interaction. SAM binds between two helical regions using predominately hydrogen bond interactions on one side and van der Waals surface complementarity on the other. In the SAM-III riboswitch (Figure 3f) [31・•], SAM is also bent, but to a lesser extent, so that the methionine moiety is placed aside of the adenine ring and is partially disordered. Therefore, unlike in SAM-I and SAM-II rboswitches, the ligand length is not critical for the SAM-III riboswitch. The adenosine moiety and the adjacent region of the methionine moiety contact RNA within a semi-open cavity formed by a junctional region. A different conformation of SAM was observed in the SAM-II 
structure (Figure 3e) [30••]. The ligand extends along the major grove face of the P2b-L1 triplex and uses all available functional groups for interactions with RNA. The hydrogen bonding patterns of SAM recognition also vary in all three riboswitches. Similarities are limited to Hoogsteen pairing with uracil and recognition of carboxylate by purines in the SAM-I and SAM-II structures. Other common features include stacking of the adenine moiety with RNA bases and, most importantly, electrostatic interactions of the positively charged sulfur moiety with $\mathrm{O} 4$ carbonyls of uracils. The readout of the positive charge is essential for discrimination between SAM and SAH, the by-product of SAM-dependent methylation reactions (Figure 2d).

\section{Specific recognition of metabolite analogues by riboswitches}

Since some riboswitches interact with metabolite-like antibiotics and contain mutations providing antibiotic resistance, riboswitch-controlled gene expression, along with other cellular processes [33], may be targeted by antimicrobial compounds [34•]. The structures of the eukaryotic TPP riboswitch with TPP and its analogues oxythiamine and pyrithiamine pyrophopshates were found to be nearly identical, with a slight conformational adjustment next to the central ring of pyrithiamine pyrophosphate [35]. No changes were identified in the structures of the lysine riboswitch with antibacterial compounds $S$-(2-aminoethyl)-L-cysteine and L-4-oxalysine (Figure 2c), while extensions in the lysine analogues L-homoarginine and N6-1-iminoethyl-L-lysine replaced water molecules and caused the rotation of RNA ribose [25••]. As in the TPP riboswitch, shortened ligands of the FMN riboswitch, riboflavin and antibiotic roseoflavin (Figure 2b), re-positioned several nucleotides in the binding pocket [27]. Additional spatial adjustment was detected in the roseoflavin-riboswitch complex for accommodation of a methyl substituent in the ligand ring system. These and earlier data indicate that the binding pockets of the TPP and FMN riboswitches $[27 \bullet \bullet, 29]$ demonstrate intrinsic plasticity, while those of purine $[7,8], g \operatorname{lm} S[12,13]$ and lysine $[25 \bullet \cdot 26 \bullet \bullet]$ riboswitches are more rigid.

\section{Riboswitch folding and function}

The folding pathway and ligand-induced conformational re-arrangements are essential aspects for understanding the molecular mechanism of the riboswitch function. Earlier experiments on purine riboswitches (Figure 4a) suggested partial organization of the sensing domain prior to metabolite binding [36-39]. Follow-up chemical probing [40,41], NMR spectroscopy [42, 43], fluorescent spectroscopy [44-47], and calorimetric studies [40] further refined the model that implies the parallel preorientation of $\mathrm{P} 2$ and $\mathrm{P} 3$ hairpins, preorganization of interacting loops L2 and L3, and local disorder of the junctional region in the absence of the ligand. To bind the junction, a purine likely samples junctional conformations until it finds a bindingcompetent form maintained by universally conserved nucleotides [40]. Ligand binding then reinforces the tertiary loop interactions [47] and stabilizes the regulatory helix P1. For effective gene control, the switching decision should be precisely timed. Since the rate of the ligandriboswitch complex formation depends on the ligand binding affinity and the kinetics of transcription [48], the regulation mechanism of related purine riboswitches with distinct expression platforms may involve different contributions from thermodynamic and kinetic components [47].

To gain further insight on the early steps of purine-riboswitch complex formation, a timeresolved NMR strategy was applied to monitor the ligand-induced folding of the guaninesensing domain [49•]. The approach is based on the laser-triggered release of hypoxanthine from the inactive photolabile 'caged' derivative in a solution of isotopically-labeled riboswitch (Figure 4b). The fast hypoxanthine binding events are then tracked by NMR spectroscopy and analyzed using molecular dynamic simulations. The experimental data suggested a three-step binding model, in which an initial fast low-affinity encounter is followed by the specific binding 
of the ligand to the riboswitch core, leading up to a final slower process that involves tightening of the tertiary structure.

In cells, riboswitch folding is tightly coupled with transcription. To obtain an integrated picture of structure formation and ligand binding after transcription, single nascent metabolite-sensing domains of adenine riboswitch were unfolded and refolded using single-molecule force spectroscopy [50••]. The assay was performed using dual-trap optical tweezers: the first trap held a transcriptionally stalled RNA polymerase, while the initial RNA transcript emerging from the polymerase was hybridized to a DNA handle in the second trap. After RNA transcription, the traps were moved apart to apply force to the RNA transcript, and the molecular extension of the transcript - related to the number of nucleotides folded - was measured as the transcript unfolded and refolded repeatedly, both in the presence and absence of the ligand. The measurements revealed sequential RNA folding (Figure 4c), which begins with formation of P2, the first fully transcribed element, followed by P3 folding. In the next adenine-competent state, the adenine binding pocket is pre-organized by tertiary contacts, sequestering the nucleotides between P2 and P3. The tertiary interactions then facilitate formation of the helix P1, the least-stable element governing conformational switching. Adenine binding stabilizes the folded state and raises the barrier for leaving the folded state; in vivo, without adenine, P1 helix formation would easily be disrupted by terminator hairpin invasion.

Folding studies on other riboswitches have proposed mechanisms of TPP riboswitch action [51-53], demonstrated the ligand-associated structural transitions in the glycine riboswitch [54•], and uncovered the importance of tertiary interactions for control by SAM-I [55] and lysine [56] riboswitches. The first ligand-free structure of the $g \operatorname{lm} S$ riboswitch/ribozyme [13] has been complemented by the structure of the unliganded lysine riboswitch $[25 \bullet \bullet, 26 \bullet \bullet]$, found to be virtually identical to the lysine-bound structure. Though the formation of the lysine-free confirmation has been facilitated by the absence of the competing anti-terminator structure and by stable crystal contacts, this result strongly supports the idea that major parts of a riboswitch can be folded prior to interaction with ligand, while ligand binding locks the conformation of key riboswitch regions and regulatory elements. In contrast to the lysine riboswitch, the similarity between ligand-bound and ligand-free forms of the $\mathrm{glmS}$ riboswitch/ribozyme is not likely caused by crystallization, since ligand binding stimulates the cleavage of the glmS RNA and does not make extensive conformational re-arrangements which, in other riboswitches, stabilize the regulatory helix P1.

\section{Conclusions}

Recent structures of the riboswitches, discussed here, have added novel junction architectures to the repertoire of RNA folds and broadened the collection of RNA structural motifs by novel variants of turns, loop-loop and loop-receptor interactions. Among the main conclusions of recent structural studies is that a variety of principles are utilized by riboswitches of different complexities to recognize their cognate ligands. Interestingly, the sizes of the riboswitches and their ligands do not correlate, suggesting that other factors, such as the number of related compounds to be discriminated against and the dynamic range of the riboswitch response, may contribute to the complexity of the riboswitch folds. A special place belongs to the SAM riboswitches that represent a striking example of convergent evolution, displaying different folds and ligand binding modes to recognize the same ligand and carry out similar regulatory functions. The lysine and FMN riboswitch structures suggest that metal ions are as important for metabolite binding as for RNA folding. However, it is still not clear whether metal ions simply expand upon a poor repertoire of functional groups in RNA to aid specific metabolite binding. It has become evident that the majority of riboswitch-bound ligands are directly involved in riboswitch function through the specific recognition of regulatory sequences or 
through participation in the tertiary interactions that facilitate formation of regulatory elements. As demonstrated by biochemical and biophysical studies, ligand binding also stabilizes partially pre-formed riboswitch architectures and contributes to the overall stability of the RNA folds. Though many challenging problems in riboswitch regulation still remain, the structural information will undoubtedly lead to a comprehensive picture of riboswitch-based gene expression control and could help in the design of tools that affect this regulation.

\section{Acknowledgments}

I thank O. Pikovskaya (Memorial Sloan-Kettering Cancer Center) for critical reading of the manuscript, and M. Woodside (University of Alberta) and J. Buck (Johann Wolfgang Goethe-University) for providing figures. This work was supported by National Institutes of Health grant GM073618.

\section{References and recommended reading}

Papers of particular interest, published within the annual period of review, have been highlighted as:

- of special interest

•• of outstanding interest

1. Nudler E, Mironov AS. The riboswitch control of bacterial metabolism. Trends Biochem Sci 2004;29:11-17. [PubMed: 14729327]

2. Winkler WC, Breaker RR. Regulation of bacterial gene expression by riboswitches. Annu Rev Microbiol 2005;59:487-517. [PubMed: 16153177]

3. Barrick JE, Breaker RR. The distributions, mechanisms, and structures of metabolite-binding riboswitches. Genome Biol 2007;8:R239. [PubMed: 17997835]

4. Henkin T. Riboswitch RNAs: using RNA to sense cellular metabolism. Genes Dev 2008;22:33833390. [PubMed: 19141470]

5. Bocobza SE, Aharoni A. Switching the light on plant riboswitches. Trends Plant Sci 2008;13:526533. [PubMed: 18778966]

6. Serganov A, Patel DJ. Ribozymes, riboswitches and beyond: regulation of gene expression without proteins. Nat Rev Genet 2007;8:776-790. [PubMed: 17846637]

7. Batey RT, Gilbert SD, Montange RK. Structure of a natural guanine-responsive riboswitch complexed with the metabolite hypoxanthine. Nature 2004;432:411-415. [PubMed: 15549109]

8. Serganov A, Yuan YR, Pikovskaya O, Polonskaia A, Malinina L, Phan AT, Hobartner C, Micura R, Breaker RR, Patel DJ. Structural basis for discriminative regulation of gene expression by adenineand guanine-sensing mRNAs. Chem Biol 2004;11:1729-1741. [PubMed: 15610857]

9. Serganov A, Polonskaia A, Phan AT, Breaker RR, Patel DJ. Structural basis for gene regulation by a thiamine pyrophosphate-sensing riboswitch. Nature 2006;441:1167-1171. [PubMed: 16728979]

10. Thore S, Leibundgut M, Ban N. Structure of the eukaryotic thiamine pyrophosphate riboswitch with its regulatory ligand. Science 2006;312:1208-1211. [PubMed: 16675665]

11. Montange RK, Batey RT. Structure of the $S$-adenosylmethionine riboswitch regulatory mRNA element. Nature 2006;441:1172-1175. [PubMed: 16810258]

12. Cochrane JC, Lipchock SV, Strobel SA. Structural investigation of the GlmS ribozyme bound to its catalytic cofactor. Chem Biol 2007;14:97-105. [PubMed: 17196404]

13. Klein DJ, Ferre-D'Amare AR. Structural basis of glmS ribozyme activation by glucosamine-6phosphate. Science 2006;313:1752-1756. [PubMed: 16990543]

14. Edwards TE, Klein DJ, Ferre-D'Amare AR. Riboswitches: small-molecule recognition by gene regulatory RNAs. Curr Opin Struct Biol 2007;17:273-279. [PubMed: 17574837]

15. Serganov A, Patel DJ. Towards deciphering the principles underlying an mRNA recognition code. Curr Opin Struct Biol 2008;18:120-129. [PubMed: 18255277] 
16••. Sudarsan N, Lee ER, Weinberg Z, Moy RH, Kim JN, Link KH, Breaker RR. Riboswitches in eubacteria sense the second messenger cyclic di-GMP. Science 2008;321:411-413. [PubMed: 18635805]Identification of the novel type of riboswitches specific to the second messenger cyclic di-GMP

17. Meyer MM, Roth A, Chervin SM, Garcia GA, Breaker RR. Confirmation of a second natural preQ $_{1}$ aptamer class in Streptococcaceae bacteria. RNA 2008;14:685-695. [PubMed: 18305186]

18• Roth A, Winkler WC, Regulski EE, Lee BW, Lim J, Jona I, Barrick JE, Ritwik A, Kim JN, Welz R, et al. A riboswitch selective for the queuosine precursor pre $\mathrm{Q}_{1}$ contains an unusually small aptamer domain. Nat Struct Mol Biol 2007;14:308-317. [PubMed: 17384645]The shortest riboswitch discovered up to date

19. Kim JN, Roth A, Breaker RR. Guanine riboswitch variants from Mesoplasma florum selectively recognize 2'-deoxyguanosine. Proc Natl Acad Sci U S A 2007;104:16092-16097. [PubMed: 17911257]

20. Wang JX, Breaker RR. Riboswitches that sense $S$-adenosylmethionine and $S$-adenosylhomocysteine. Biochem Cell Biol 2008;86:157-168. [PubMed: 18443629]

21. Wang JX, Lee ER, Morales DR, Lim J, Breaker RR. Riboswitches that sense $S$-adenosylhomocysteine and activate genes involved in coenzyme recycling. Mol Cell 2008;29:691-702. [PubMed: 18374645]

22. Cromie MJ, Shi Y, Latifi T, Groisman EA. An RNA sensor for intracellular $\mathrm{Mg}^{2+}$ Cell 2006;125:7184. [PubMed: 16615891]

23••. Dann CE 3rd, Wakeman CA, Sieling CL, Baker SC, Irnov I, Winkler WC. Structure and mechanism of a metal-sensing regulatory RNA. Cell 2007;130:878-892. [PubMed: 17803910]Identification of a novel $\mathrm{Mg}^{2+}$ sensor and the first structure of a metal-sensing regulatory RNA

24. Regulski EE, Moy RH, Weinberg Z, Barrick JE, Yao Z, Ruzzo WL, Breaker RR. A widespread riboswitch candidate that controls bacterial genes involved in molybdenum cofactor and tungsten cofactor metabolism. Mol Microbiol 2008;68:918-932. [PubMed: 18363797]

25••. Serganov A, Huang L, Patel DJ. Structural insights into amino acid binding and gene control by a lysine riboswitch. Nature 2008;455:1263-1267. [PubMed: 18784651]Describes the high-resolution structure of the lysine-bound riboswitch, as well as the riboswitch structures in the ligand-free state and bound to lysine analogues. Demonstrates the importance of the $\mathrm{K}^{+}$cation for lysine binding

26••. Garst AD, Heroux A, Rambo RP, Batey RT. Crystal structure of the lysine riboswitch regulatory mRNA element. J Biol Chem 2008;283:22347-22351. [PubMed: 18593706]Along with [25], crystal structures of the lysine riboswitch in the ligand-bound and ligand-free states

27••. Serganov A, Huang L, Patel DJ. Coenzyme recognition and gene regulation by a flavin mononucleotide riboswitch. Nature 2009;458:233-237. [PubMed: 19169240]Describes the FMN riboswitch structure and uncovers molecular recognition principles of FMN binding by various techniques

28•. Jaeger L, Verzemnieks EJ, Geary C. The UA_handle: a versatile submotif in stable RNA architectures. Nucleic Acids Res 2009;37:215-230. [PubMed: 19036788]Identification of a versatile motif present in structured RNAs. The paper correctly predicted architectural elements in the FMN riboswitch

29. Edwards TE, Ferre-D'Amare AR. Crystal structures of the thi-box riboswitch bound to thiamine pyrophosphate analogs reveal adaptive RNA-small molecule recognition. Structure 2006;14:14591468. [PubMed: 16962976]

30••. Gilbert SD, Rambo RP, Van Tyne D, Batey RT. Structure of the SAM-II riboswitch bound to $S$ adenosylmethionine. Nat Struct Mol Biol 2008;15:177-182. [PubMed: 18204466]Presents the crystal structure and biochemical characterization of the SAM-II riboswitch

31••. Lu C, Smith AM, Fuchs RT, Ding F, Rajashankar K, Henkin TM, Ke A. Crystal structures of the SAM-III/S $\mathrm{S}_{\mathrm{MK}}$ riboswitch reveal the SAM-dependent translation inhibition mechanism. Nat Struct Mol Biol 2008;15:1076-1083. [PubMed: 18806797]Crystal structure and biochemical experiments on the SAM-III riboswitch

32. Yamauchi T, Miyoshi D, Kubodera T, Nishimura A, Nakai S, Sugimoto N. Roles of $\mathrm{Mg}^{2+}$ in TPPdependent riboswitch. FEBS Lett 2005;579:2583-2588. [PubMed: 15862294] 
33. Ataide SF, Wilson SN, Dang S, Rogers TE, Roy B, Banerjee R, Henkin TM, Ibba M. Mechanisms of resistance to an amino acid antibiotic that targets translation. ACS Chem Biol 2007;2:819-827. [PubMed: 18154269]

34•. Blount KF, Breaker RR. Riboswitches as antibacterial drug targets. Nat Biotechnol 2006;24:15581564. [PubMed: 17160062]An interesting discussion on the potential utilization of riboswitches as antibiotic targets

35. Thore S, Frick C, Ban N. Structural basis of thiamine pyrophosphate analogues binding to the eukaryotic riboswitch. J Am Chem Soc 2008;130:8116-8117. [PubMed: 18533652]

36. Gilbert SD, Stoddard CD, Wise SJ, Batey RT. Thermodynamic and kinetic characterization of ligand binding to the purine riboswitch aptamer domain. J Mol Biol 2006;359:754-768. [PubMed: 16650860]

37. Lemay JF, Penedo JC, Tremblay R, Lilley DM, Lafontaine DA. Folding of the adenine riboswitch. Chem Biol 2006;13:857-868. [PubMed: 16931335]

38. Mandal M, Boese B, Barrick JE, Winkler WC, Breaker RR. Riboswitches control fundamental biochemical pathways in Bacillus subtilis and other bacteria. Cell 2003;113:577-586. [PubMed: 12787499]

39. Noeske J, Buck J, Furtig B, Nasiri HR, Schwalbe H, Wohnert J. Interplay of 'induced fit' and preorganization in the ligand induced folding of the aptamer domain of the guanine binding riboswitch. Nucleic Acids Res 2007;35:572-583. [PubMed: 17175531]

40. Gilbert SD, Love CE, Edwards AL, Batey RT. Mutational analysis of the purine riboswitch aptamer domain. Biochemistry 2007;46:13297-13309. [PubMed: 17960911]

41. Stoddard CD, Gilbert SD, Batey RT. Ligand-dependent folding of the three-way junction in the purine riboswitch. RNA 2008;14:675-684. [PubMed: 18268025]

42. Noeske J, Schwalbe H, Wohnert J. Metal-ion binding and metal-ion induced folding of the adeninesensing riboswitch aptamer domain. Nucleic Acids Res 2007;35:5262-5273. [PubMed: 17686787]

43. Ottink OM, Rampersad SM, Tessari M, Zaman GJ, Heus HA, Wijmenga SS. Ligand-induced folding of the guanine-sensing riboswitch is controlled by a combined predetermined induced fit mechanism. RNA 2007;13:2202-2212. [PubMed: 17959930]

44. Eskandari S, Prychyna O, Leung J, Avdic D, O’Neill MA. Ligand-directed dynamics of adenine riboswitch conformers. J Am Chem Soc 2007;129:11308-11309. [PubMed: 17713907]

45. Lemay JF, Lafontaine DA. Core requirements of the adenine riboswitch aptamer for ligand binding. RNA 2007;13:339-350. [PubMed: 17200422]

46. Mulhbacher J, Lafontaine DA. Ligand recognition determinants of guanine riboswitches. Nucleic Acids Res 2007;35:5568-5580. [PubMed: 17704135]

47. Rieder R, Lang K, Graber D, Micura R. Ligand-induced folding of the adenosine deaminase Ariboswitch and implications on riboswitch translational control. Chembiochem 2007;8:896-902. [PubMed: 17440909]

48. Wickiser JK, Cheah MT, Breaker RR, Crothers DM. The kinetics of ligand binding by an adeninesensing riboswitch. Biochemistry 2005;44:13404-13414. [PubMed: 16201765]

49•. Buck J, Furtig B, Noeske J, Wohnert J, Schwalbe H. Time-resolved NMR methods resolving ligandinduced RNA folding at atomic resolution. Proc Natl Acad Sci U S A 2007;104:15699-15704. [PubMed: 17895388]An elegant study of guanine riboswitch folding using photolabile riboswitch ligand

50••. Greenleaf WJ, Frieda KL, Foster DA, Woodside MT, Block SM. Direct observation of hierarchical folding in single riboswitch aptamers. Science 2008;319:630-633. [PubMed: 18174398]An integrated view on adenine riboswitch folding using single-molecule force spectroscopy

51. Lang K, Rieder R, Micura R. Ligand-induced folding of the thiM TPP riboswitch investigated by a structure-based fluorescence spectroscopic approach. Nucleic Acids Res 2007;35:5370-5378. [PubMed: 17693433]

52. Ontiveros-Palacios N, Smith AM, Grundy FJ, Soberon M, Henkin TM, Miranda-Rios J. Molecular basis of gene regulation by the THI-box riboswitch. Mol Microbiol 2008;67:793-803. [PubMed: 18179415] 
53. Rentmeister A, Mayer G, Kuhn N, Famulok M. Conformational changes in the expression domain of the Escherichia coli thiM riboswitch. Nucleic Acids Res 2007;35:3713-3722. [PubMed: 17517779]

54•. Lipfert J, Das R, Chu VB, Kudaravalli M, Boyd N, Herschlag D, Doniach S. Structural transitions and thermodynamics of a glycine-dependent riboswitch from Vibrio cholerae. J Mol Biol 2007;365:1393-1406. [PubMed: 17118400]Low resolution small-angle X-ray scattering structures of the glycine riboswitch in different states

55. Heppell B, Lafontaine DA. Folding of the SAM aptamer is determined by the formation of a K-turndependent pseudoknot. Biochemistry 2008;47:1490-1499. [PubMed: 18205390]

56. Blouin S, Lafontaine DA. A loop loop interaction and a K-turn motif located in the lysine aptamer domain are important for the riboswitch gene regulation control. RNA 2007;13:1256-1267.

[PubMed: 17585050] 
(a)

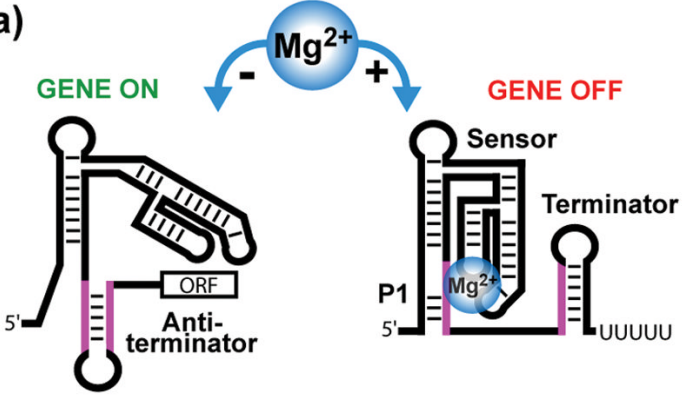

(c)

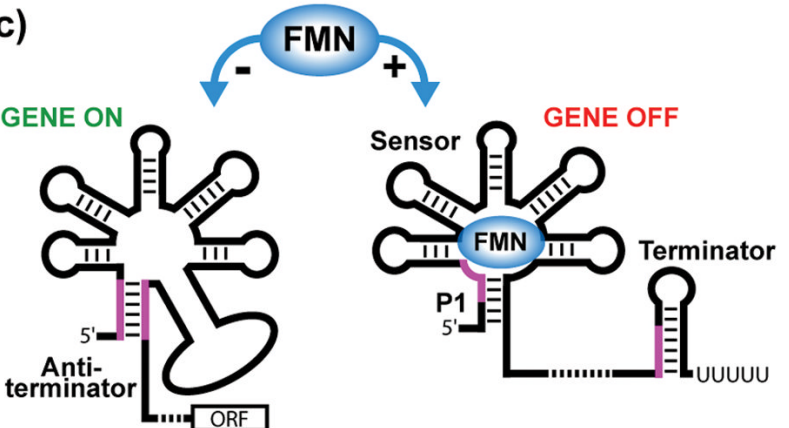

(b)

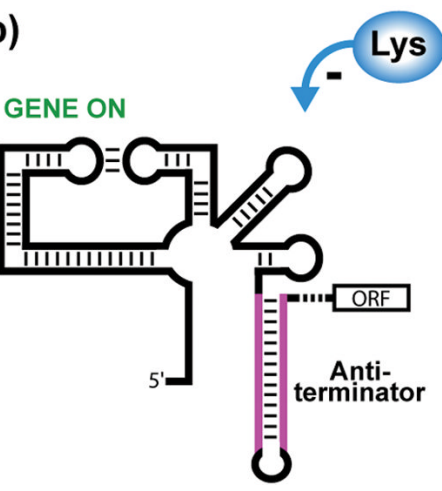

(d)

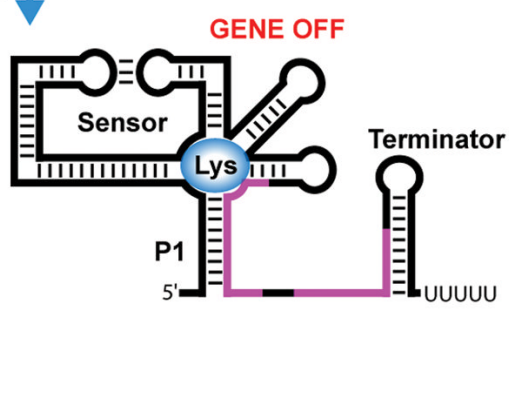

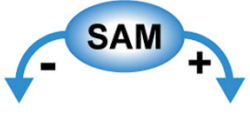

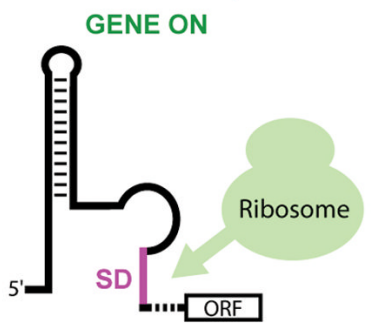

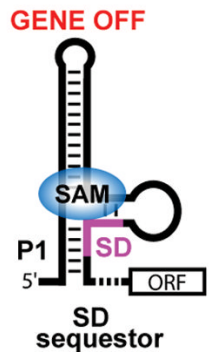

Figure 1.

Gene expression control by various riboswitches. Transcriptional attenuation mechanism in the M-box $\mathrm{Mg}^{2+}$ sensor (a), lysine riboswitch (b) and flavin mononucleotide (FMN) riboswitch (c). In the absence of ligand, mRNA forms an anti-terminator helix and transcription can proceed through the open reading frame (ORF). In the presence of ligand, the sensing domain binds the cognate metabolite, thereby stabilizing the P1 helix, triggering formation of a transcription terminator in the expression platform and turning off expression of the downstream gene. Complementary anti-terminator sequences are shown in magenta. (d), Regulation of translation initiation by the type III $S$-adenosylmethionine (SAM) riboswitch. Without ligand, Shine-Dalgarno (SD) sequence is accessible for ribosome entry and translation initiation. In the presence of SAM, SD sequence is paired, thereby preventing ribosome binding. 
(a)

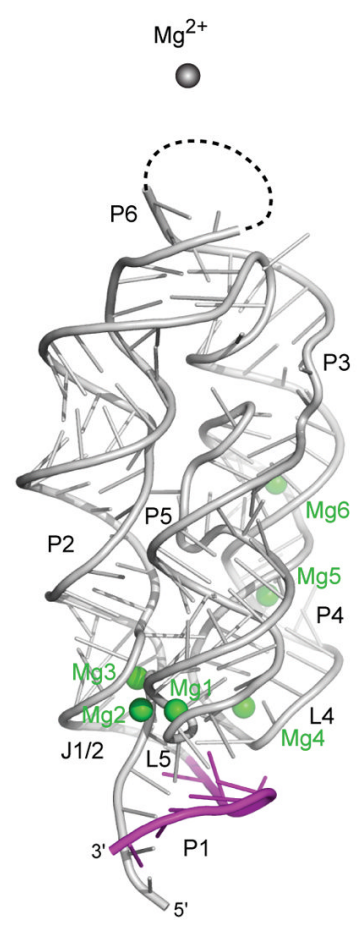

(b)
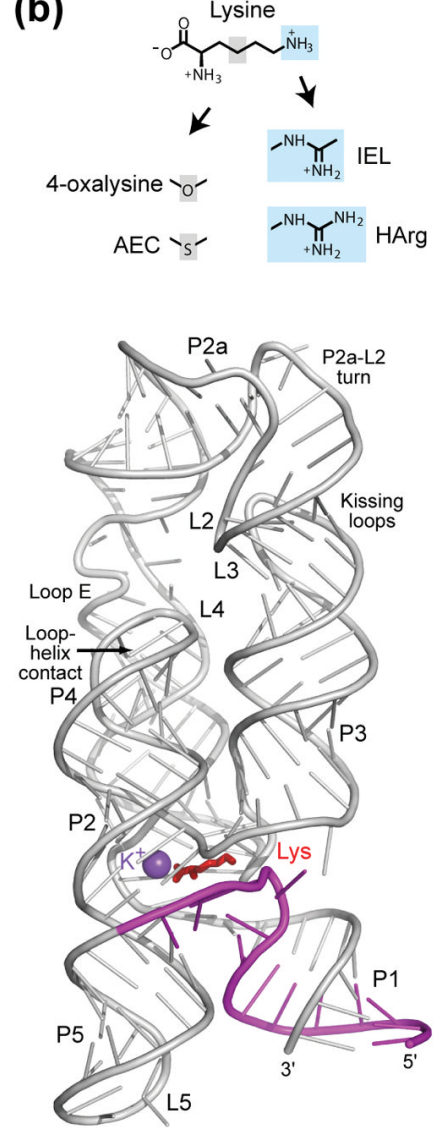

(c)

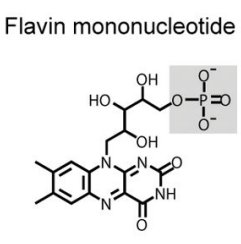

Riboflavin

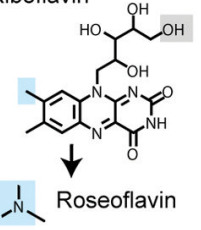

P2

P2

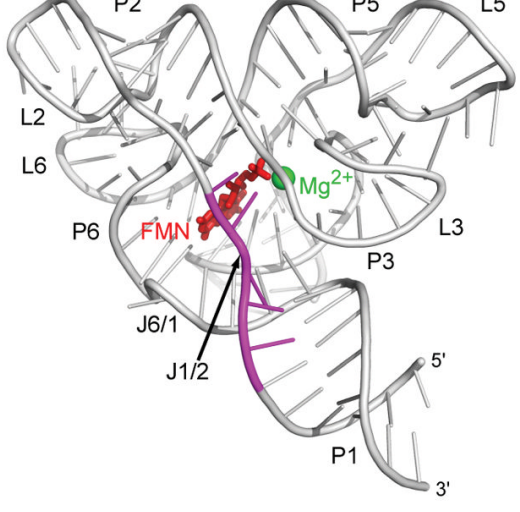

(d)
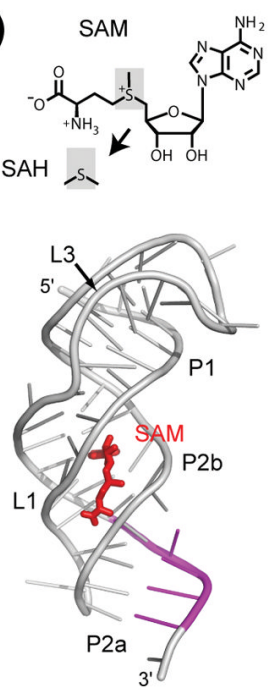

(e)

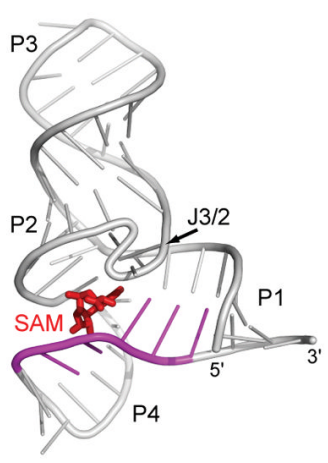

Figure 2.

Tertiary structures of ligand-bound riboswitches and chemical structures of riboswitch ligands. Metabolites are in red. RNA segments in magenta indicate regulatory or 'switching' sequences (a-c) that participate in formation of helix P1 or anti-terminator. Grey and blue shading highlights changes in the compounds. (a), M-box $\mathrm{Mg}^{2+}$ sensor. Six $\mathrm{Mg}^{2+}$ cations are depicted as green spheres. (b), Lysine-sensing domain, the cognate ligand lysine and riboswitch-binding derivatives of lysine. AEC, $S$-(2-aminoethyl)-L-cysteine; IEL, N6-1-iminoethyl-L-lysine; HArg, L-homoarginine. Potassium cation is in violet. (c), FMN-sensing domain, its cognate ligand FMN, the FMN precursor riboflavin, and antibiotic roseoflavin. (d), Type II $S$ adenosylmethionine (SAM) riboswitch and the cognate ligand SAM. $S$-adenosylhomocysteine (SAH) lacks a methyl group (highlighted) and does not bind the riboswitch strongly. SD sequence is indicated in magenta. (e), SAM-III riboswitch. 
(a) $\mathrm{Mg}^{2+}$

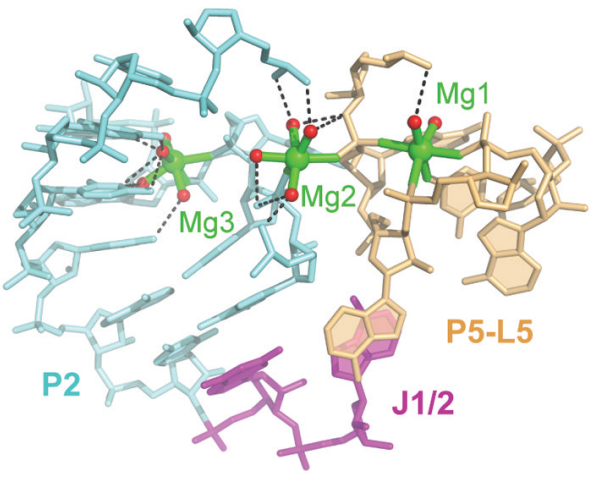

(d)
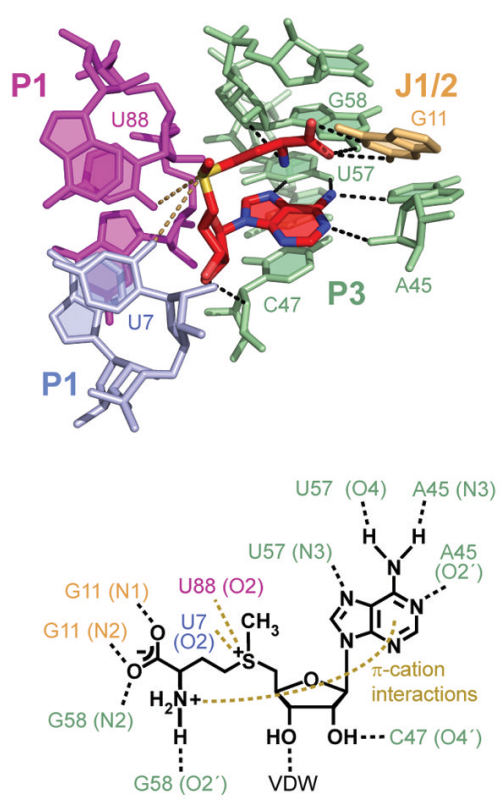

(b)

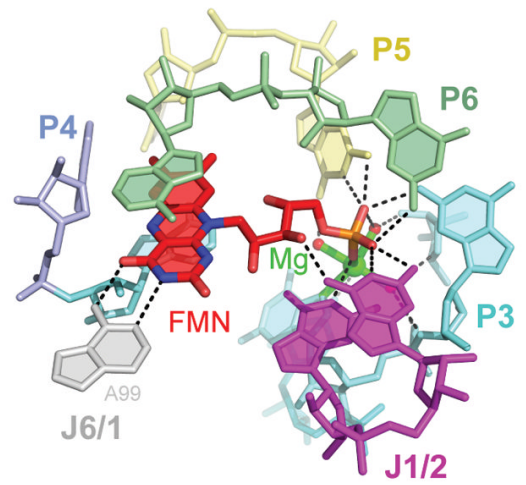

(e)
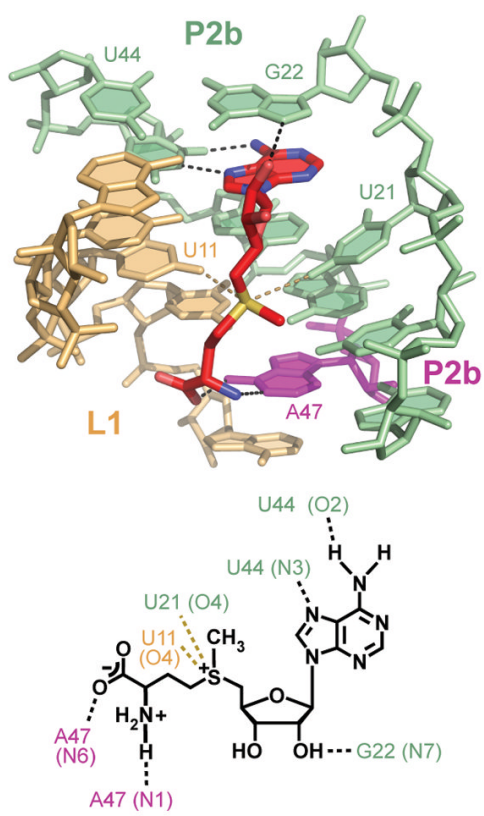

(c)

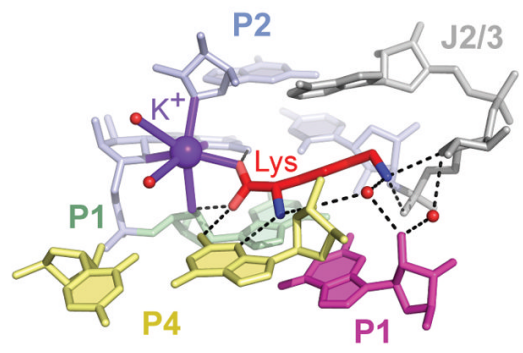

(f)

SAM-III
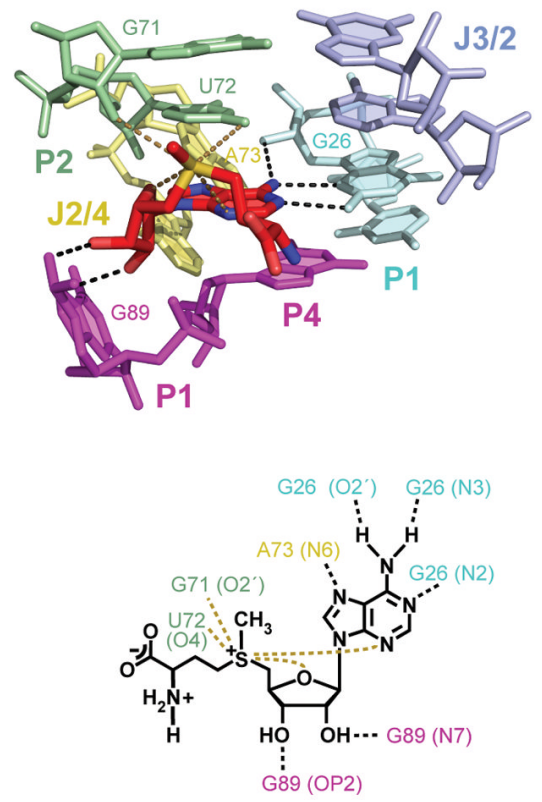

Figure 3.

Ligand recognition by riboswitches. Magenta color depicts nucleotides of the regulatory RNA segment, as in Figure 2. Other nucleotides are colored according with the RNA elements. Hydrogen bonds and hydrogen bond distances are shown with black dashed lines. (a) M-box $\mathrm{Mg}^{2+}$ sensor. $\mathrm{Mg}^{2+}$ cations Mg1-3 and water molecules are shown as green and red spheres, respectively. $\mathrm{Mg}^{2+}$ coordination bonds are depicted by green sticks. (b), FMN-sensing domain. (c), Lysine-sensing domain. $\mathrm{K}^{+}$cation is shown as a violet sphere, while its coordination bonds are depicted by violet sticks. (d), SAM-I riboswitch (top) and schematic representation of SAM recognition (bottom) according with [20]. VDW, van der Waals interactions. Electrostatic interactions are shown with gold dashed lines. (e), SAM-II riboswitch. (f), SAM-III riboswitch. 


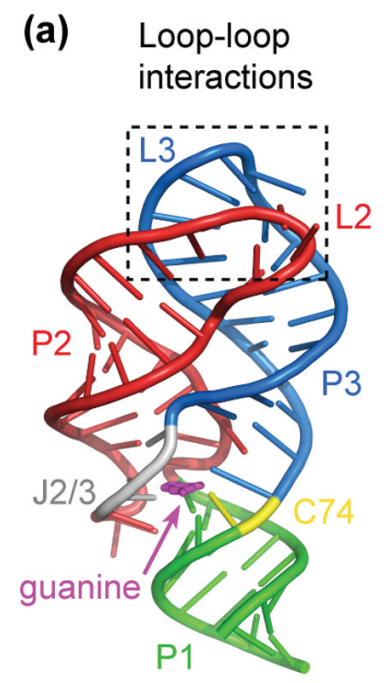

(b)

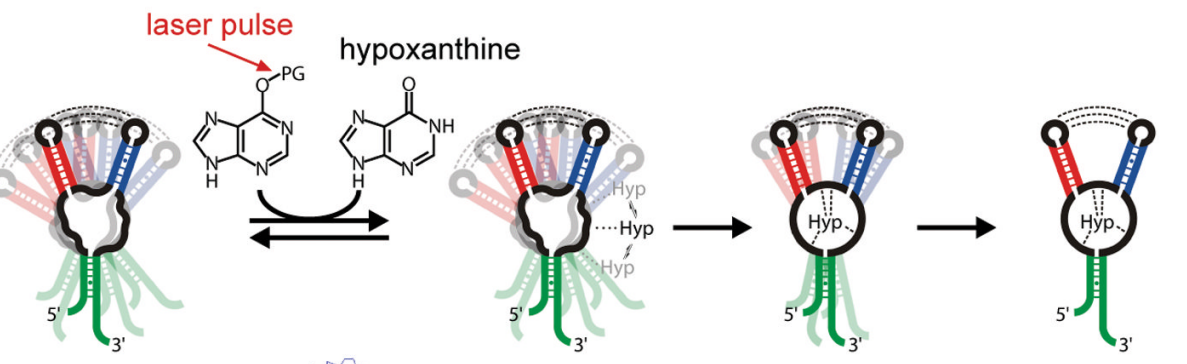

(c)

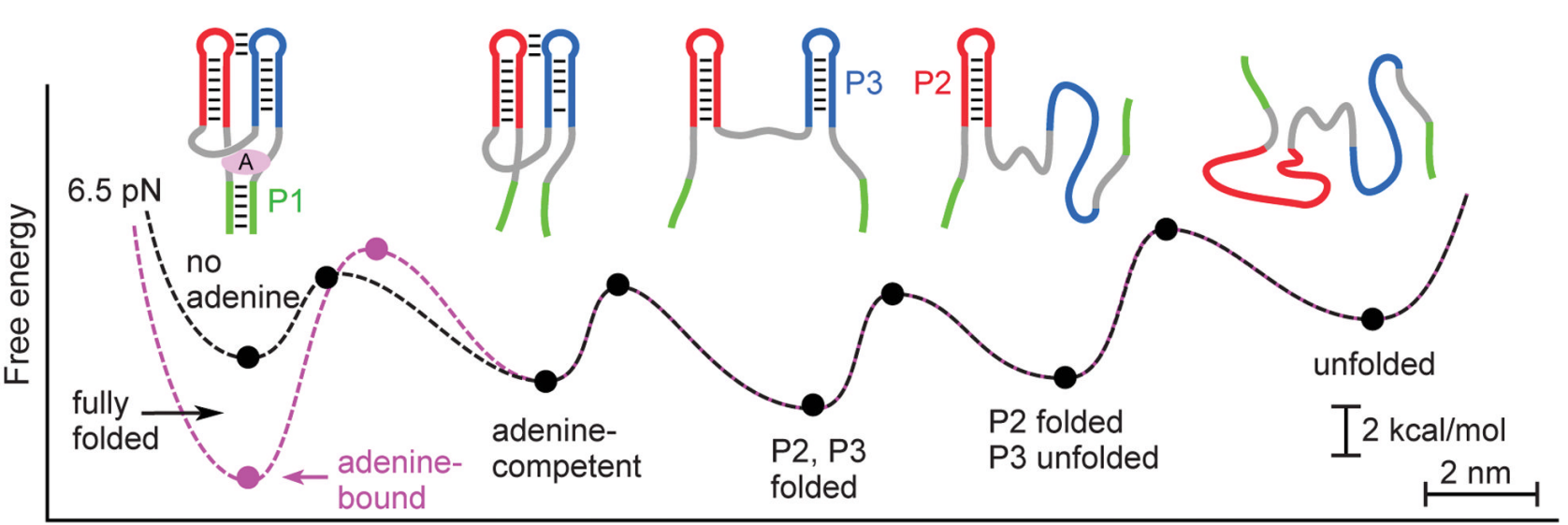

Extension

Figure 4.

Folding of purine riboswitches. (a) Crystal structure of the guanine-sensing riboswitch bound to guanine [8]. Discriminatory nucleotide C74 that forms a Watson-Crick base pair with bound guanine is in yellow. In the similar adenine riboswitch, this nucleotide is replaced by uracil.

(b) Folding of the guanine riboswitch in the presence of photolabile hypoxanthine derivative [49•]. The three-step folding model based on the experimentally restrained torsion angle molecular dynamics simulations is shown on top. Overlaid structures of the three states simulated according with NMR data, aligned on P2, are shown below. Adapted from Fig. 4a, b in [49•] (copyright 2007 National Academy of Sciences USA). (c) Folding of the adeninesensing riboswitch domain studied using single-molecule force spectroscopy. Energy landscapes are shown for the folding reactions in the presence (red) and absence (black) of adenine $[50 \bullet \bullet$. 